\title{
CHARACTERIZATIONS OF THE SPACE OF CONTINUOUS FUNCTIONS OVER A COMPACT HAUSDORFF SPACE
}

\author{
BY \\ R. F. ARENS AND J. L. KELLEY
}

1. This paper consists of two parts, in each of which is established a completely characteristic property of those Banach spaces which are, as Banach spaces, the class of all continuous, bounded, real-valued functions over some completely regular topological space.

The first characterization proceeds via an investigation of the "extreme points" (\$3) of the unit sphere $\Sigma$ of the adjoint space $B^{*}$ of the Banach space $B$ under consideration. If $B$ is the class of continuous real-valued functions over a compact Hausdorff space $X$, the norm $\|f\|$ of any $f$ in $B$ is defined by

$$
\|f\|=\sup _{x \in X}|f(x)|
$$

(in this case we write $B=C_{X}$ ), then the class of extreme point of $\Sigma$ can be divided into two disjoint closed sets each of which is homeomorphic to $X$, using the weak topology in $B^{*}$. In the proof we use the Riesz-Markoff-Saks representation $[10]\left({ }^{1}\right)$ for linear continuous functionals on $C$. Our first characterization is the converse of this: if we have a Banach space $B$ such that the extreme points of $\Sigma$ in $B^{*}$ can be divided into two classes each lying in a plane supporting $\Sigma$, and if any class of extreme points of $\Sigma$ having no antipodal limit points always lies in some plane supporting $\Sigma$, then $B$ is $C_{X}$ for some $X$.

The second characterization involves properties of the collection of maximal convex subsets of the surface of the sphere of the Banach space under consideration. This mode of characterization is a natural extension of the methods used by M. H. Stone $[11$, p. 469] and by S. Eilenberg [4] in generalizing Banach's theorem [2, p. 170]. This theorem, that two compact Hausdorff spaces are homeomorphic if and only if $C_{X}$ and $C_{Y}$ are equivalent, is an immediate inference from either of the two investigations we make. In the first case a new proof of the theorem results, but in the second case the proof differs from known proofs only in the method of introducing the topology in the "reconstructed" space.

The present characterizations are wholly in terms of objects and operations defined in all Banach spaces. Previous characterizations of the spaces $C$ are not strictly of this nature: thus Stone $[12 ; 13]$ singles out certain properties of lattice-ordered rings and groups. which are characteristic of $C_{\mathbf{X}}$;

Presented to the Society, August 20, 1946; received by the editors November 30, 1946.

(1) Numbers in brackets refer to the bibliography at the end of the paper. 
I. Gelfand [5] bases his representation on suitable Banach algebra conditions; S. Kakutani-[6] has shown how to represent a certain kind of Banach lattice; S. Krein and M. Krein [7] proceed from a characteristic property of the set of all non-negative functions in $C_{x}$. Mr. J. A. Clarkson and Mr. S. M. Myers have communicated to us that they have obtained characterizations involving only Banach space notions.

2. A lemma on function spaces. Any Banach space is equivalent to a subspace of $C_{X}$ for some $X$. It is important in our later work to have a criterion which will decide whether a subspace of $C_{X}$ is all of $C_{X}$. Such a criterion is the following.

2.1 Lemma. Let $Y$ be a dense subset of $X$, a compact Hausdorff space, and let $B$ be a complete linear subspace of $C_{X}$. If for every pair of subsets $K$ and $L$ of $Y$ whose closures'are disjoint there is an $f \in B$, with $0 \leqq f(x) \leqq 1$ for all $x \in X$, $f(x)=1$ for $x \in K, f(x)=0$ for $x \in L$, then $B=C_{X}$.

Since the presence of the constant functions is assured (one sets $K=Y$ ) any two distinct numbers can be used instead of 0 and 1.

Proof. We shall show $B$ to be dense in $C_{X}$, and this together with the completeness of $B$ will prove the lemma.

Let $F \in C_{X}$ and $e>0$ be given. Choose a real number $a$ and an integer $n$ such that

$$
a \leqq F(x) \leqq n e+a \quad \text { (for all } x \in X) .
$$

For each $m, 0 \leqq m \leqq n$, let

$$
\begin{aligned}
& L_{m}=\{x \mid F(x) \leqq(m-1) e+a\}, \\
& K_{m}=\{x \mid F(x) \geqq m e+a\} .
\end{aligned}
$$

(We use " $\{x \mid \ldots\}$ " for "the set of $x$ such that $\ldots$. ") The closures of $K_{m}$ and $L_{m}$ are surely disjoint. Let $f_{m} \in B$ be the functions hypothesized by the lemma, and let

$$
f=a f_{0}+e\left(f_{1}+\cdots+f_{n}\right) .
$$

Then $f \in B$. For any $x \in X$, for some $m$,

$$
a+(m-1) e \leqq F(x) \leqq a+m e .
$$

Then $x \in K_{m-1} \supset K_{m-2} \supset \cdots$ and $x \in L_{m+1} \subset L_{m+2} \subset \cdots$, and hence

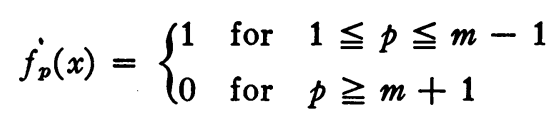

and $f(x)=a+e(m-1)+e f_{m}(x)$. Thus

$$
a+(m-1) e \leqq f(x) \leqq a+m e \text { and }|F(x)-f(x)| \leqq e .
$$


The lemma is thus established.

3. Topology in $B^{*}$. Extreme points. Suppose $\Sigma$ is any subset of a topological linear space $L$.

An extreme point [8] $\xi$ of $\Sigma$ is a point through which one cannot pass a segment lying in the set $\Sigma$ and having both ends distinct from $\xi$.

Let $M$ be a closed, linear, proper subspace of $L$, having the property that it is properly contained in any closed linear subspace of $L$ except $L$ itself. Let $\xi_{0}$ be an element of $L$. Then we shall call the set $H=\xi_{0}+M$ of elements $\xi=\xi_{0}+\zeta, \zeta \in M$, a hyperplane, or briefly, a plane in $L$. (Cf. [2, p. 246].)

A plane $H$ in $L$ is called a plane supporting a set $\Sigma$ if it contains limit elements of that set, and if whenever it intersects the interior of any segment whose end points lie in $\Sigma$, then these end points are in $H$ as well.

Consider now the adjoint $B^{*}$ of a given Banach space $B$, which consists of all the linear continuous functionals defined on $B$. For each $\xi \in B^{*}$ one customarily introduces the notation

$$
\|\xi\|=\sup _{\|f\|=1}|\xi(f)|
$$

We use it mainly to single out the unit sphere $\Sigma$ of $B^{*}$, consisting of those $\xi$ for which $\|\xi\| \leqq 1$. However, when we speak of limits in $B^{*}$ we have in mind the familiar "weak" topology in $B^{*}$, which we shall denote by " $w^{*}$-topology." It may be defined as follows [1]: A directed set $\xi_{\nu}$ in $B^{*}$ is said to converge to $\xi$ if and only if the directed set of real numbers $\xi_{\nu}(f)$ converges to $\xi(f)$ for every $f$ in $B$.

In consequence of the definition of the $w^{*}$-topology, all hyperplanes have the form given by an equation

$$
\xi(f)=c
$$

where $f$ and $c$ are determined by the hyperplane in question. If a hyperplane is a plane of support of the unit sphere $\Sigma$ then it can evidently be written in the form

$$
\xi(f)=1 \quad(\|f\|)=1) .
$$

We preface our first theorem in this section by remarking that the class of extreme points of $\Sigma$ is known to be nonvoid [8] and that $\Sigma$ is in fact the closed convex hull of its class of extreme points.

3.1 Lemma. The mapping $x \rightarrow x^{*}$, where $x^{*}(f)=f(x)$ for $x \in X$ and $f \in C_{X}$, is an embedding of $X$ into the class of extreme points of $\Sigma, C_{X}^{*}$ having the $w^{*}$-topology. The image $X^{*}$ of $X$ under $x \rightarrow x^{*}$ and its diametric set $-X^{*}$ have no common limit point on $\Sigma$.

Proof. Suppose $x_{0} \in X$, and that $\xi=x_{0}^{*}$ is not an extreme point. Then there are two distinct elements $\eta_{1}, \eta_{2} \in \Sigma$ such that $2 x_{0}^{*}=\eta_{1}+\eta_{2}$. There exist two 
unique completely additive regular set functions $\mu_{1}, \mu_{2}$, defined for Borel sets of $X$, having the properties [6]:

$$
\eta_{i}(f)=\int_{X} f(x) \mu_{i}(d x) \quad(i=1,2),
$$

the integration being in the Lebesgue-Stieltjes sense; and

$$
\left\|\eta_{i}\right\|=\text { the total variation of } \mu_{i} \text { on } X \text {, }
$$

the total variation over a set $E \subset X$ being defined

$$
W\left(\mu_{i}, E\right)=\sup \mu_{i}\left(E_{1}\right)-\inf \mu_{i}\left(E_{2}\right),
$$

$E_{1}$ and $E_{2}$ ranging independently over the Borel subsets of $E . W\left(\mu_{i} ;\right)$ is also a completely additive set function [9].

Since $\eta_{1} \neq x_{0}^{*} \neq \eta_{2}$, there must be a neighborhood $V$ of $x_{0}$ such that $W\left(\mu_{i} ; V\right)<1(i=1,2)$, otherwise a contradiction is quickly obtainable. There then exists an $f \in B,\|f\|=1, f\left(x_{0}\right)=1$, and $f(x)=0$ if $x \in V$.

From the representations (1) of $\eta_{i}(f)$ and familiar estimates of bounds on integrals, we obtain $\eta_{1}(f)<1$ and $\eta_{2}(f)<1$. This contradicts $\eta_{1}+\eta_{2}=2 x_{0}^{*}$ and $x_{0}^{*}(f)=f\left(x_{0}\right)=1$. Thus $x_{0}^{*}$ and $-x_{0}^{*}$ are extreme points of $\Sigma$.

Now the mappings $x \rightarrow x^{*}$ and $x \rightarrow-x^{*}$ are each obviously 1-to-1, and in view of the definition of $w^{*}$-topology are readily seen to be homeomorphisms. Thus the first statement of the lemma is proved.

Let $u \in C_{X}$ be the function which is identically 1 on $X$. It is easily seen that $X^{*}$ lies on the plane $\xi(u)=1$ and $-X^{*}$ lies on the plane $\xi(u)=-1$, and that these planes do not intersect. Thus the sets $X^{*}$ and $-X^{*}$ have no common limit points, as was also to be shown.

The converse of this lemma is the following.

3.2 Lemma. If $\xi$ is an extreme point of $\Sigma$ in $C_{X}^{*}$ then there is a point $x$ of $X$ such that either $\xi(f)=f(x)$ for all $f$ or $\xi(f)=-f(x)$ for all $f$.

Proof. Suppose $\xi$ is an extreme point of $\Sigma$ and there is no $x \in X$ such that $\xi= \pm x^{*}$. Clearly $\|\xi\|=1$. The compactness of $X$ readily enables one to find two disjoint open sets $V_{1}, V_{2}$ in $X$ such that $0<W_{i}<1, i=1,2$, where $W_{i}=W\left(\mu, V_{i}\right)$ and $\mu$ is the set-function corresponding to $\xi$.

In the subsequent discussion we shall replace $\int f(x) \mu(d x)$ by $\int$ alone.

Now define $\eta$ by

$$
\eta(f)=W_{1} \int_{V_{2}}-W_{2} \int_{V_{1}}
$$

Clearly $\eta \in B^{*}$, and a consideration of the set function corresponding to $\eta$ shows that $\|\eta\|=2 W_{1} W_{2}>0$. Next define $\zeta_{1}=\xi+\eta, \zeta_{2}=\xi-\eta$. Then $\zeta_{1}, \zeta_{2} \in B^{*}$, and $2 \xi=\zeta_{1}+\zeta_{2}$. 
This argument will produce a contradiction by showing that $\xi$ is not an extreme point, for we shall show that $\zeta_{1}$ and $\zeta_{2}$ belong to the set $\Sigma$.

Consider $\zeta_{1}$, and an $f \in B$ for which $\|f\| \leqq 1$; we have

$$
\begin{aligned}
\zeta_{1}(f) & =\int_{X}+W_{1} \int_{V_{2}}-W_{2} \int_{V_{1}} \\
& =\int_{X-V_{1}-V_{2}}+\left(1+W_{1}\right) \int_{V_{2}}+\left(1-W_{2}\right) \int_{V_{1}} .
\end{aligned}
$$

Using our information on the variation of $\mu$, we can estimate

$$
\left|\zeta_{1}(f)\right| \leqq 1-W_{1}-W_{2}+\left(1+W_{1}\right) W_{2}+\left(1-W_{2}\right) W_{1}=1
$$

whence $\left\|\zeta_{1}\right\| \leqq 1$ and $\zeta_{1}, \zeta_{2} \in \Sigma$. Since this is a contradiction, the conclusion of this lemma follows.

We note that the Banach-Stone uniqueness theorem $[2$, p. $170 ; 1$, p. 469] follows from the preceding results:

3.3 Theorem (cf. [11, p. 469]). If $X$ and $Y$ are compact Hausdorff spaces, and $C_{X}$ and $C_{Y}$ are equivalent, then $X$ and $Y$ are homeomorphic.

Proof. If $C_{X}$ and $C_{Y}$ are equivalent, that is to say, linearly isomorphic under a norm-preserving correspondence, then the adjoints $C_{\bar{X}}^{*}$ and $C_{Y}^{*}$ are also equivalent under a homeomorphism. Thus $Y^{*}$ can be homeomorphically transferred from the unit sphere of $C_{Y}^{*}$ into the union on $X^{*}$ and $-X^{*}$, and that part of $Y^{*}$ which falls into $-X^{*}$ must obviously have no limit points in common with the rest of $Y^{*}$, and hence its image in $-X^{*}$ may be reflected through the origin in $C_{X}^{*}$ onto the uncovered part of $X^{*}$. Thus $X^{*}$ and $Y^{*}$, and finally $X$ and $Y$, are homeomorphic.

4. First characterization, by means of $B^{*}$. With the aid of the preceding results, we can establish our first main theorem.

4.1 Theorem. $A$ Banach space $B$ is the space of continuous real-valued functions on a compact Hausdorff space if and only if the unit sphere $\Sigma$ of the conjugate space $B^{*}$ with $w^{*}$-topology is such that:

(1) There are two supporting planes of $\Sigma$ which together contain all the extreme points of $\Sigma$.

(2) Any collection of extreme points of $\Sigma$ whose closure contains no antipodal points lies entirely in some plane supporting $\Sigma$.

Proof of necessity. Let $B=C_{x}$.

Condition (1) has been established in the last paragraph of the proof of 3.1 , the planes of support in question being defined by the equations

$$
\xi(u)=+1 \text { and } \xi(u)=-1 ; \quad \xi \in B^{*} .
$$

Let $T$ be a collection of extreme points whose closure has no antipodal 
pair of points. Let $K$ be the subset of $T$ lying in the plane $\xi(u)=1$, and let $-L$ be the part in the plane $\xi(u)=-1$. Then $K$ and $L$ are images of two sets of $X$ whose closures do not intersect. Since $X$ is normal, $f \in B$ exists which has the value 1 on the former, the value -1 on the latter, and intermediate values elsewhere. Therefore all extreme points lie in the region $R$ defined by $-1 \leqq \xi(f) \leqq 1$. We conclude that $\Sigma \subset R$ and that the plane $\xi(f)=1$ supports $\Sigma$.

Proof of sufficiency. Suppose an arbitrary Banach space $B$ satisfies the conditions of 4.1. Let $X_{1}$ be the set of extreme points of $\Sigma$ lying in one of the planes of support required by (1), and let $X_{2}$ be the set lying in the other. $X_{1}$ and $X_{2}$ clearly have no common limit points and are diametric to each other. Let $X$ be the closure of one of them, say $X_{1}$, in the $w^{*}$-topology. Since $\Sigma$ is a compact Hausdorff space [1, p. 255], $X$ is a compact Hausdorff space.

Any element $f \in B$ determines a continuous real-valued function over $X$, if we write $f(\xi)=\xi(f)$. Since $\Sigma$ is the closed convex hull of its extreme points, we can readily infer that

$$
\sup _{\xi \in \Sigma}|f(\xi)|=\sup _{\xi \in X}|f(\xi)| .
$$

Since the former of these is well known to have the value $\|f\|$, it appears that $B$ is normed as a function space over $X$.

Now let $K, L$ be subsets of $X_{1}$ without common limit point. Then the points of $K,-L$ do not have any antipodal limit points. Hence by (2), for some $f \in B, f(\xi)=1$ for $\xi \in K \cup-L$, while $-1 \leqq f(\xi) \leqq 1$ for all other $\xi \in \Sigma$. Clearly, $\xi \in K$ implies $f(\xi)=1$, while $\xi \in L$ implies $f(\xi)=-1$. Since $X_{1}$ is dense in $X$, an appeal to Lemma 2.1 finishes the proof.

It is interesting to note that $X_{1}$ and $X$ coincide, as a consequence of the proof; whence the set $X_{1}$ was closed in the $w^{*}$-topology. There is no reason to suppose that the set of extreme points of the unit sphere in the adjoint $B^{*}$ of an arbitrary Banach space $B$ is a closed set in the $w^{*}$-topology.

5. Maximal convex subsets of the sphere surface. In this section we prove certain geometric results about $C_{X}$, most of which are already known. These results furnish the necessary conditions for the characterization of $C_{X}$, to be given in the following section.

Let $B$ be a Banach space and let $E$ denote its unit sphere, $E=\{f \mid f \in B$ and $\|f\| \leqq 1\}$. Let $S$ be the surface of the unit sphere, $S=\{f \mid f \in B$ and $\|f\|=1\}$. If $B=C_{X}, X$ a compact Hausdorff space, then an element $f \in E$ is also an element of $S$ if and only if $|f(x)|=1$ for some $x \in X$.

We now have:

5.1 Lemma. Suppose $B=C_{X}$. Then the convex set spanned by a set $f_{1}, \cdots, f_{n}$ of elements of $S$ is contained in $S$ if and only if an $x$ in $X$ can be found such that $f_{1}(x)=f_{2}(x)=\cdots=f_{n}(x)=\dot{e}$ where $e= \pm 1$.

Proof. The sufficiency being clear, we consider the necessity. Suppose 
$\lambda_{1}, \cdots, \lambda_{n} \geqq 0, \lambda_{1}+\cdots+\lambda_{n}=1$ so that $\lambda_{1} f_{1}+\ldots+\lambda_{n} f_{n}=f$ lies in the convex hull of $\left\{f_{1}, \cdots, f_{n}\right\}$, and suppose for each $x \in X$ one of the numbers $\left\{f(x), \cdots, f_{n}(x)\right\}$ is not equal to $e,|e|=1$. By the compactness of $X$ one can readily infer that

$$
\|f\|=\sup _{x \in X}|f(x)|<1
$$

whence $f \in E$ but $f \notin S$.

The same argument as that just presented also demonstrates:

5.2 If $f, g \in S \subset C_{X}$ then for any element $h$ interior to the line segment joining $f$ and $g$ the subset of $X$ on which h assumes the value $e,|e|=1$, is the intersection of the corresponding sets for $f$ and $g$.

Hence, we have the following lemma.

5.3 Lemma. If $B=C_{X}, f \in B$ is an extreme point of $E$ if and only if $|f(x)|=1$ for all $x \in X$.

We now consider the geometry of the "faces" of $E$.

5.4 Definition. If $B$ is a Banach space, $\mathcal{C}(B)$ is the collection of maximal convex subsets of the surface of the unit sphere.

5.5 Lemma. If $B=C_{X}$ then $C \in \mathcal{C}(B)$ if and only if for some $x \in X$, and for some $e,|e|=1, C=\{f \mid f \in B,\|f\|=1$, and $f(x)=e\}$.

Proof. If $C$ is a convex subset of $S$ then for either $e=+1$ or $e=-1$ it must be true, by Lemma 5.1 , that for any finite set $f_{1}, \cdots, f_{n} \in C$ the set $\{x \mid x \in X$, $f_{i}(x)=e$ for each $\left.i=1, \cdots, n\right\}$ is closed and nonvoid. Since $X$ is compact, and since this statement says that the family of sets of the form $\{x \mid x \in X$, $f(x)=e\}$, where $f \subset C$, has the finite intersection property, we have $C \subset\{f \mid f \in B$ and $f(x)=e\}$ for some $x \in X$. On the other hand any set $\{f \mid f \in B$ and $f(x)=e\}$ is itself convex. This proves the lemma.

5.6. Lemma. If $B=C_{X}$ any set $C \in \mathcal{C}(B)$ together with its opposite spans the unit sphere in the sense that for $f \in E$ there are $g, h \in C$ and $\lambda, 0 \leqq \lambda \leqq 1$, such that $f=\lambda g-(1-\lambda) h$.

Proof. From 5.3, $C$ is of the form, for some $x_{0} \in X$ and some $e,|e|=1$, $\left\{g \mid g \in B\right.$ and $\left.g\left(x_{0}\right)=e\right\}$. We may suppose $e=1$. Let $v=f\left(x_{0}\right)$. If $|v| \neq 1$ (otherwise the lemma is proved), let

$$
p(x)=\max \left\{\frac{1-f(x)}{1-v}, \frac{1+f(x)}{1+v}\right\} .
$$

Let $g=f+(1-v) p$ and $h=-f+(1+v) p$. It is a straightforward calculation to show $g, h \in C$ and $f=(v+1) g / 2+(v-1) h / 2$. Any convex subset of $S$ which spans $E$ in the sense of the above lemma defines a linear function as follows. 
5.7 Lemma. If the convex set CCS spans the unit sphere $E$ of a Banach space $B$ then there is a unique $\xi_{c} \in B^{*},\left\|\xi_{c}\right\|=1$, which on $C$ has the value 1 . For $f \in E, \xi_{c}(f)=1-d(f, C)$, where $d(f, C)=\inf _{o \in c}\|f-g\|$.

Proof. The existence of one linear function $\xi_{c}$ is an easy consequence of the Hahn-Banach theorem. The uniqueness follows from the spanning condition. If $f \in E, f=\lambda g-(1-\lambda) h$ where $0 \leqq \lambda \leqq 1$ and $g, h \in C$ then $\xi_{C}(f)=\lambda$ $-(1-\lambda)=2 \lambda-1$. Now $d(f, C) \leqq\|h-f\|=\|(1-\lambda) h+(1-\lambda) g\|=(1-\lambda)\|h+g\|$ $=2(1-\lambda)=1-\xi_{C}(f)$. On the other hand, since $\left\|\xi_{c}\right\|=1, d(f, C)=\inf _{o \in c}\|g-f\|$ $\geqq \inf _{o \in C}\left|\xi_{C}(g-f)\right|=1-\xi_{C}(f)$, and the lemma is proved.

The Banach-Stone theorem may now be stated as follows:

5.8 Theorem. Let $B=C_{x}$, and let $u$ be a fixed extreme point of the unit sphere of $B$. Let $\mathcal{C}_{1}$ be the collection of all maximal convex subsets of the sphere surface to which $u$ belongs. If $X^{*}$ is the set of all linear functions $\xi_{C}$ which on $C \in C_{1}$ have the value 1 , then $X^{*}$ in the $w^{*}$-topology is homeomorphic to $X$.

Hence $C_{X}$ is equivalent to $C_{Y}$ if and only if $X$ is homeomorphic to $Y$.

Proof. From 5.3 and 5.5, for each $C \in \mathcal{C}_{1}$ there is some $x_{C} \in X$ such that $C=\left\{f \mid f\left(x_{C}\right)=u\left(x_{C}\right)\right\}$. This point $x_{C}$ is unique, and the function $\xi_{C}$ which has the value one on $C$ is also unique. A one-to-one correspondence is thus established between $X$ and $X^{*}$. It is easy to see that $\xi_{C}(f)=f\left(x_{C}\right) u\left(x_{C}\right)$ since for $f \in C$ both have the value 1. Finally, if $x_{\alpha}$ is a directed set, $x_{\alpha} \rightarrow x$ if and only if for each $f, f\left(x_{\alpha}\right) u\left(x_{\alpha}\right) \rightarrow f(x) u(x)$, and the theorem is proved.

There is one more property of spaces of the type $C_{X}$ which will be utilized in the following section.

5.10 Lemma. If $A \subset \mathcal{C}\left(C_{X}\right)$ and the intersection of the elements of $\mathcal{A}$ is void then there are directed sets $C_{\beta}^{\prime}$ and $C_{\beta}^{\prime \prime}$ in $A$ and $d\left(f, C_{\beta}^{\prime}\right)+d\left(f, C_{\beta}^{\prime \prime}\right)^{\prime} \rightarrow 2$ for all $f \in E$.

Proof. Write $\mathcal{A}$ as the union of $\mathcal{A}^{+}$and $\mathcal{A}^{-}$, where $C \in \mathcal{A}^{+}$if for some $x \in X, C=\left\{f \mid f \in C_{X},\|f\|=1\right.$ and $\left.f(x)=+1\right\}$. Let $A^{+}$be the set of points of $X$ determined by $\mathcal{A}^{+}$and let $A^{-}$be the corresponding set for $\mathcal{A}^{-}$. If there were a continuous function, of norm 1 , on $X$ with value +1 on $A^{+}$and value -1 on $A^{-}$this function would be an element common to each set of the collection $\mathcal{A}$. Hence there must be a point $x$ which is either a point or limit point of both $A^{+}$and $A^{-}$. Therefore there are directed sets $x_{\beta}^{\prime} \in A^{+}, x_{\beta}^{\prime \prime} \in A^{-}$and $x_{\beta}^{\prime} \rightarrow x, x_{\beta}^{\prime \prime \prime} \rightarrow x$. Writing $C_{\beta}^{\prime}=\left\{f \mid f \in C_{X},\|f\|=1\right.$, and $\left.f\left(x_{\beta}^{\prime}\right)=+1\right\}, C_{\beta}^{\prime \prime}=\{f \mid f$ $\in C_{X},\|f\|=1$ and $\left.f\left(x_{\beta}^{\prime \prime}\right)=-1\right\}$ it is seen that the conclusion of the lemma holds.

6. Second characterization, by means of $B$. Half of the following theorem has now been established.

6.1. Theorem. A Banach space $B$ is equivalent to the space of continuous real-valued functions over some compact Hausdorff space if and only if the follow- 
ing conditions hold.

(a) The unit sphere of $B$ has an extreme point.

(b) If $C$ is a maximal convex subset of the surface of the unit sphere then $C$ and the set symmetric to $C$ with respect to 0 span the unit sphere.

(c) If there is no element common to all members of a collection $A$ of maximal convex subsets of the surface of the unit sphere then there are directed sets $C_{\beta}^{\prime}$, $C_{\beta}^{\prime} \in \mathcal{C}$ such that

$$
d\left(f, C_{\beta}^{\prime}\right)+d\left(f, C_{\beta}^{\prime \prime}\right) \rightarrow 2
$$

for all $f$ for which $\|f\| \leqq 1$.

Proof. Suppose $B$ has the properties (a), (b) and (c). Let $u$ be a fixed extreme point of $E$, and let $\mathcal{C}_{1}$ be the set of elements of $\mathcal{C}(B)$ to which $u$ belongs. In view of the spanning property (b) each element of $\mathcal{C}(B)$ must contain $u$ or $-u$. Now let $Y$ be the set of all elements $\xi_{c} \in B^{*}$, of norm 1, having on $C$ the value one, where $C \in \mathcal{C}_{1}$. (See 5.8.) We assert that $B$ is equivalent to $C_{X}$, where $X$ is the closure of $Y$ in $B^{*}$.

First, each $f \in B$ corresponds to a function of $f^{\prime} \in C_{X}$ by means of the definition

$$
f^{\prime}\left(\xi_{C}\right)=\xi_{C}(f)
$$

This correspondence actually establishes an equivalence between $B$ and a subspace of $C_{X}$. This will be established if it is shown that $\|f\|=\sup _{\xi \in X}|\xi(f)|$. Since, for $\xi \in X,\|\xi\| \leqq 1$, surely $\sup _{\xi \in X}|\xi(f)| \leqq\|f\|$. On the other hand, if $f \neq 0, f /\|f\|$ belongs to some $C \in \mathcal{C}$, and either $f /\|f\|$ or $-f /\|f\|$ belongs to some $C \in \mathcal{C}_{1}$. For the function $\xi_{C}$ corresponding to this $C,\left|\xi_{C}(f)\right|=\|f\|$. The equivalence of $B$ to a subspace of $C_{X}$ is thus established.

It remains to be shown that $B$ is equivalent to all of $C_{X}$. To this end, using the lemma of $\S 2$, it must be shown that if $K, L \subset Y$, the closure of $K$ being disjoint from that of $L$, there is an $f \in B$, with $\|f\|=1, f$ being +1 on $K$ and -1 on $L$. (To be precise, $\xi(f)=+1$ for $\xi \in K$ and -1 for $\xi \in \dot{L}$.) Let $\mathcal{A}$ be the subcollection consisting of elements corresponding to $\xi \in K$, or corresponding to $\xi$, with $-\xi \in L$. There is an $f,\|f\|=1$, with value +1 on $K$ and -1 on $L$ if and only if the intersection of all the elements of $\mathcal{A}$ is nonvoid, for any $f$ lying in the intersection has precisely the desired properties. Assume the intersection is void. Then, by the hypothesis (c), there are directed sets $C_{\beta}^{\prime}, C_{\beta}^{\prime \prime} \in \mathcal{A}$ with $d\left(f, C_{\beta}^{\prime}\right)+d\left(f, C_{\beta}^{\prime \prime}\right) \rightarrow 2$ for all $f \in E$. The corresponding functions, $\xi_{\beta}^{\prime}$ and $\xi_{\beta}^{\prime \prime}$, by Lemma 5.8, have the property that $\xi_{\beta}^{\prime}(f)+\xi_{\beta}^{\prime \prime}(f) \rightarrow 0$ for all $f \in E$. In particular, this convergence must hold for $u$, and $\left|\xi_{\beta}^{\prime}(u)\right|$ $=\left|\xi_{\beta}^{\prime \prime}(u)\right|=1$. Therefore, for a cofinal subset we must have $\xi_{\beta}^{\prime} \in K$ and $-\xi_{\beta}^{\prime \prime} \in L$ or else $\xi_{\beta}^{\prime \prime} \in K$ and $-\xi_{\beta}^{\prime} \in L$. There is a cluster point $\xi$ of $\xi_{\beta}^{\prime}$, since the unit sphere in $B^{*}$ is compact in the $w^{*}$-topology. Since $\xi_{\beta}^{\prime}(f)+\xi_{\beta}^{\prime \prime}(f) \rightarrow 0$ for all $f \in E, \xi$ is also a cluster point of the set of all $-\xi_{\beta}^{\prime \prime}$. Hence $K$ and $L$ have a common limit point, contrary to hypothesis. The intersection of the elements of $\mathcal{A}$ is hence nonvoid, and the theorem is proved. 


\section{BIBLIOGRAPHY}

1. L. Alaoglu, Weak topologies of normed linear spaces, Ann. of Math. vol. 41 (1940) pp. 252-267.

2. S. Banach, Théorie des opérations linéaires, Warsaw, 1932.

3. H. F. Bohnenblust and S. Kakutani, Concrete representation of (M)-spaces, Ann. of Math. vol. 42 (1941) pp. 1025-1028. 579.

4. S. Eilenberg, Banach space methods in topology, Ann. of Math. vol. 43 (1942) pp. 568-

5. I. Gelfand, On normed rings, C. R. (Doklady) Acad. Sci. URSS. vol. 23 (1939) pp. 430432.

6. S. Kakutani, Concrete representation of abstract (M)-spaces, Ann. of Math. vol. 42 (1941) pp. 994-1024.

7. S. Krein and M. Krein, On an inner characteristic of the set of all continuous functions defined on a bicompact Hausdorff space, C. R. (Doklady) Acad. Sci. URSS. vol. 27 (1940) pp. 427-430.

8. M. Krein and D. Milman, On the extreme points of regularly convex sets, Studia Mathematica vol. 9 (1940) pp. 133-138.

9. S. Saks, Theory of the integral, Warsaw-Lw6w, 1937.

10. - Integration in abstract metric spaces, Duke Math. J. vol. 4 (1938) pp. 408-411.

11. M. H. Stone, Applications of the theory of Boolean rings to general topology, Trans. Amer. Math. Soc. vol. 41 (1937) pp. 375-481.

12. - A general theory of spectra, Proc. Nat. Acad. Sci. U.S.A. vol. 26 (1940) pp. 280-283.

13. — A general theory of spectra, II, Proc. Nat. Acad. Sci. U.S.A. vol. 27 (1941) pp. 83-87.

Institute for Advanced Study,

Princeton, N. J.

University of Chicago,

Chicago, Ill. 